\title{
CENTRAL SEPARABLE ALGEBRAS WHICH ARE LOCALLY ENDOMORPHISM RINGS OF FREE MODULES
}

\author{
BERNICE L. AUSLANDER
}

\begin{abstract}
The object of this paper is to study the kernel of the map of the Brauer group of an integrally closed noetherian domain $A$ into the direct product of the Brauer groups of the localizations of $A$ at prime ideals. It is shown that this kernel is isomorphically contained in the torsion subgroup of the first cohomology group of the sheaf of Cartier divisors over Spec $A$. As a consequence, the author describes several new sets of conditions on $A$ which guarantee that the kernel is trivial.
\end{abstract}

We recall that $M$. Auslander and O. Goldman [2] define the Brauer group of a commutative ring $A$ as follows: let $\mathfrak{A}(A)$ be the isomorphism classes of central separable $A$-algebras, and $\mathfrak{A}_{0}(A)$ the subset of $\mathfrak{U}(A)$ consisting of endomorphism rings of finitely generated projective faithful $A$-modules. Let $\mathfrak{R}_{1}$ and $\mathfrak{R}_{2}$ in $\mathfrak{A}(A)$ be equivalent if there are algebras $\Re_{1}$ and $\Re_{2}$ in $\mathfrak{U}_{0}(A)$ such that $\Re_{1} \otimes_{A} \Re_{1}$ is isomorphic to $\mathfrak{R}_{2} \otimes_{A} \mathfrak{R}_{2}$. Then $\mathfrak{B}(A)$, the set of equivalence classes of $\mathfrak{A}(A)$ under this relation, is a group, the Brauer group of $A$. If $S$ is a commutative $A$-algebra, then there is a homomorphism from $\mathfrak{B}(A)$ to $\mathfrak{B}(S)$ reduced by the operation $\ell \rightarrow S \oplus_{A} \mathfrak{l}$. In particular, for each prime ideal $\mathfrak{p}$ of $A$ there is a homomorphism from $\mathfrak{B}(A)$ to $\mathfrak{B}\left(A_{\mathfrak{p}}\right)$. Thus we have a homomorphism $\theta$ from $\mathfrak{B}(A)$ to $\Pi_{\mathfrak{p}} \mathfrak{B}\left(A_{\mathfrak{p}}\right)$, where $\mathfrak{p}$ ranges over the prime ideals of $A$, defined by mapping the class of $\mathfrak{R}$ (which we will also denote $\mathfrak{R}$ without fear of confusion), to $\left\{\mathfrak{R}_{\mathfrak{p}}\right\}$. We would like to examine the kernel of this map in the case where $A$ is an integrally closed noetherian domain.

We are particularly interested in knowing when this kernel is trivial. In other words, we would like to answer the following question: given a central separable $A$-algebra $L$ such that for every prime ideal $\mathfrak{p}$ of $A$ the localization $L_{\mathfrak{p}}$ is isomorphic to $\operatorname{Hom}_{A_{\mathfrak{p}}}\left(F_{\mathfrak{p}}, F_{\mathfrak{p}}\right)$ for some

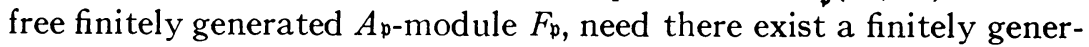
ated projective $A$-module $P$ such that $L$ is isomorphic to $\operatorname{Hom}_{A}(P, P)$ ? According to results known at this time, it may be that the answer to

Received by the editors October 28, 1969.

AMS 1970 subject classifications. Primary 13A20.

Key words and phrases. Integrally closed noetherian domain, localization at prime ideals, prime spectrum, sheaf of Cartier divisors, semilocal domain, local unique factorization domain. 
this question is yes for any integrally closed noetherian domain $A$; in other words, that the kernel is always trivial for such a ring. In a certain number of special cases this is known to be true. It is true for example where $A$ is a local domain, where $A$ is a regular domain [2, Theorem 7.2], and where $A$ is a local unique factorization domain with the property that every separable extension is a unique factorization domain [1, Corollary VII.13]. The first result is trivial, since if $\mathrm{m}$ is the maximal ideal of $A$, then $A_{\mathfrak{m}}=A$. The latter two results follow directly from the fact that in each case $\mathfrak{B}(A) \subset \mathfrak{B}(K)$ where $K$ is the field of quotients of $A$.

We will show that for any integrally closed noetherian domain $A$, the kernel of $\theta$ is isomorphically contained in the torsion subgroup of the first cohomology group of the sheaf of Cartier divisors over Spec $A$. As a consequence, we will find several new sets of conditions on $A$ which guarantee that $\theta$ is a monomorphism.

Let $M(A)$ be the set of finitely generated reflexive $A$-modules $M$ such that the localization $M_{\mathfrak{p}}$ is isomorphic to $I^{\mathfrak{p}} \otimes_{A_{\mathfrak{p}}} F_{\mathfrak{p}}$ for each prime ideal $\mathfrak{p}$ of $A$, where $I^{\mathfrak{p}}$ is a reflexive ideal in $A_{\mathfrak{p}}$ and $F_{\mathfrak{p}}$ is a free finitely

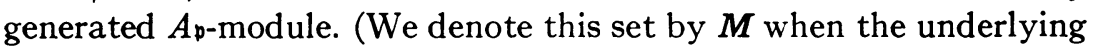
ring is unambiguous.) It is easily verified that $M$ is a commutative monoid under the operation $M \circ N=\left(M \otimes_{A} N\right)^{* *}$ (the double dual of $M \otimes N)$.

Let $P(A)$ be the submonoid of $M(A)$ consisting of finitely generated projective $A$-modules. Again, we denote this by $P$ where there is no danger of confusion. For $M_{1}$ and $M_{2}$ in $M$, we define $M_{1}$ to be equivalent to $M_{2}$ if and only if there exist modules $N_{1}$ and $N_{2}$ in $P$ such that $M_{1} \circ N_{1} \approx M_{2} \circ N_{2}$. Then $M / P$, the set of equivalence classes thus defined, is a group under the operation inherited from $M$. We will verify the presence of inverses. If $M$ is in $M$, then $\operatorname{Hom}_{\Lambda}(M, A)$ is also in $M$, for

$$
\begin{aligned}
\left.\operatorname{Hom}_{\boldsymbol{A}}(M, A)\right)_{\mathfrak{p}} & =\operatorname{Hom}_{\boldsymbol{A}_{\mathfrak{p}}}\left(M_{\mathfrak{p}}, A_{\mathfrak{p}}\right) \approx \operatorname{Hom}_{\boldsymbol{A}_{\mathfrak{p}}}\left(I^{\mathfrak{p}} \otimes F_{\boldsymbol{A}_{\mathfrak{p}}}, A_{\mathfrak{p}}\right) \\
& \approx \operatorname{Hom}_{\boldsymbol{A}_{\mathfrak{p}}}\left(I^{\mathfrak{p}}, A_{\mathfrak{p}}\right) \otimes F_{\mathfrak{p}} .
\end{aligned}
$$

Now $\mathrm{Hom}_{A_{\mathfrak{p}}}\left(I^{\mathfrak{p}}, A_{\mathfrak{p}}\right)$ is isomorphic to an ideal of $A_{\mathfrak{p}}$, and is reflexive since $I^{\mathfrak{p}}$ is. Furthermore, since $M$ is reflexive, $\left(M \otimes_{A} \operatorname{Hom}_{A}(M, A)\right)^{* *}$ $\approx \operatorname{Hom}_{A}(M, M)$. But $\operatorname{Hom}_{A}(M, M)$ is projective, for

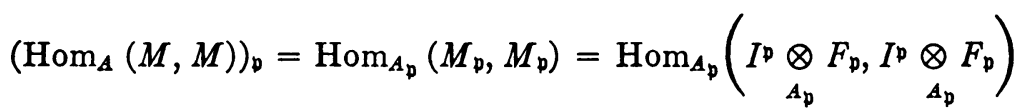

$$
\begin{aligned}
& \approx \operatorname{Hom}_{A_{\mathfrak{p}}}\left(I^{\mathfrak{p}}, I^{\mathfrak{p}}\right) \otimes \underset{\boldsymbol{A}_{\mathfrak{p}}}{\otimes} F_{\mathfrak{p}} \otimes F_{A_{\mathfrak{p}}} F_{\mathfrak{p} .}
\end{aligned}
$$


Now $\operatorname{Hom}_{A_{\mathfrak{p}}}\left(I^{\mathfrak{p}}, I^{\mathfrak{p}}\right) \approx A_{\mathfrak{p}}$, since $A_{\mathfrak{p}}$ is an integrally closed noetherian domain. Thus $\left(\operatorname{Hom}_{\boldsymbol{A}}(M, M)\right)_{\mathfrak{p}}$ is free for each prime ideal $\mathfrak{p}$, and so $\operatorname{Hom}_{\Lambda}(M, M)$ is projective. Therefore $\left[\left(M \otimes_{A} \operatorname{Hom}_{A}(M, A)\right)^{* *}\right]$ is the identity class in $M / P$, so that the inverse of the class of $M$ is the class of $\operatorname{Hom}_{\boldsymbol{A}}(M, A)$.

Let $W(A)$ be the set of finitely generated reflexive $A$-submodules of $K$, the field of quotients of $A$ (i.e. reflexive fractionary ideals). If $M$ is in $W$, then $M$ is itself isomorphic to a reflexive ideal of $A$, and so $M$ is in $M . W$ is a submonoid of $\boldsymbol{M}$, and is in fact a group under the operation since if $M$ is in $W$, so is $\operatorname{Hom}_{A}(M, A)$, and $\left(\operatorname{Hom}_{A}(M, A) \otimes_{A} M\right)^{* *} \approx \operatorname{Hom}_{A}(M, M) \approx A$. It is known that $W$ is isomorphic to the free abelian group generated by the minimal primes of $A$, the group of divisors. The isomorphism is defined as follows: since for every minimal prime ideal $\mathfrak{p}$ of $A, A_{\mathfrak{p}}$ is a discrete valuation ring and hence a principal ideal domain, for every $M$ in $W$, $M_{\mathfrak{p}}$ is principal as an $A_{\mathfrak{p}}$-module. Given a minimal prime $\mathfrak{p}$, let $x_{\mathfrak{p}}$ be a generator of $M_{\mathfrak{p}}$ over $A_{\mathfrak{p}}$. Then we map $M$ into $\bigoplus_{\mathfrak{p} \text { minimal }}$ op $\left(x_{\mathfrak{p}}\right) \cdot \mathfrak{p}$, where $v_{p}$ is the $p$-adic valuation on $K$. This sum is independent of the choice of generator, since if $y_{\mathfrak{p}}$ also generates $M_{\mathfrak{p}}$ over $A_{\mathfrak{p}}, v_{\mathfrak{p}}\left(y_{\mathfrak{p}}\right)$ $=v_{p}\left(x_{p}\right)[5$, no. 3 , Theorem 2].

Let $\boldsymbol{C}$ be the subgroup of $W$ consisting of finitely generated projective $A$-submodules of $K$. This subgroup is known as the group of Cartier divisors. Then we have the monoid diagram

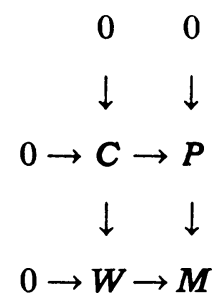

giving rise to the induced monomorphism of groups $0 \rightarrow W / C \rightarrow M / P$. We can also define a homomorphism of $M / P \rightarrow \mathfrak{B}(A)$ which sends $[M]$ in $M / P$ into $\operatorname{Hom}_{A}(M, M)$ in $\mathfrak{B}(A)$. To check that $\operatorname{Hom}_{A}(M, M)$ is indeed an element of $\mathfrak{B}(A)$ we note that the localization $\left(\operatorname{Hom}_{\mathcal{A}}(M, M)\right)_{\mathfrak{p}}$ is separable over $A_{\mathfrak{p}}$ for every minimal prime ideal $\mathfrak{p}$ of $A$. Furthermore, as was seen above, $\operatorname{Hom}_{A}(M, M)$ is projective as an $A$-module and hence [2, Proposition 4.6] it is a separable $A$ algebra. If $M$ is in $P$ then $\operatorname{Hom}_{A}(M, M)$ is trivial in $\mathscr{B}(A)$, so the map is well-defined. Thus we may construct the sequence of abelian groups

$$
0 \rightarrow W / C \rightarrow M / P \rightarrow \mathfrak{B}(A) \rightarrow \Pi \mathfrak{B}\left(A_{\mathfrak{p}}\right) .
$$


THEOREM 1. The sequence $0 \rightarrow W / C \rightarrow M / P \rightarrow \mathfrak{B}(A) \rightarrow \Pi \mathfrak{B}\left(A_{\mathfrak{b}}\right)$ is exact.

Proof. $[M]$ in $M / P$ is mapped to $\{0\}$ in $\mathscr{B}(A)$ if and only if $\operatorname{Hom}_{A}(M, M) \approx \operatorname{Hom}_{A}(P, P)$ where $P$ is some finitely generated projective $A$-module. This implies (as may be deduced from [3, Proposition A.6]) that $M \approx I \otimes_{A} P$ where $I$ is a reflexive ideal in $A$ isomorphic to $\operatorname{Hom}_{R}(P, M)$ for $R=\operatorname{Hom}_{A}(P, P)$ (i.e., $\left.I \in W\right)$. But this means that $[M]=[I]$ in $M / P$, or that $[M]$ comes from $W / C$.

Furthermore, $\mathfrak{R}$ in $\mathfrak{B}(A)$ is mapped to $\{0\}$ in $\Pi \mathfrak{B}\left(A_{\mathfrak{p}}\right)$ if and only if $\mathfrak{R}_{\mathfrak{p}} \approx \operatorname{Hom}_{A_{\mathfrak{p}}}\left(F_{\mathfrak{p}}, F_{\mathfrak{p}}\right)$ for each prime ideal $\mathfrak{p}$, where $F_{\mathfrak{p}}$ is a free finitely generated $A_{p}$-module. Thus it is clear that if $[M]$ is in $M / P$ then the image of $[M]$ in $\Pi \mathscr{B}\left(A_{\mathfrak{p}}\right)$, which is $\left\{\operatorname{Hom}_{A_{\mathfrak{p}}}\left(M_{\mathfrak{p}}, M_{\mathfrak{p}}\right)\right\}$, is trivial because of the way in which $M$ was defined. On the other hand, if $\mathfrak{l}_{\mathfrak{p}}$ is trivial in $\mathfrak{B}\left(A_{\mathfrak{p}}\right)$ for each $\mathfrak{p}$, it is certainly true that $\mathfrak{Q} \otimes_{\mathbb{A}} K$ is trivial in $\mathfrak{B}(K)$; i.e., $\mathfrak{R} \otimes_{A} K \approx \operatorname{Hom}_{K}(V, V)$ for $V$ a finite-dimensional vector space over $K$. Then since $\mathfrak{R}$ is a maximal order in $\mathfrak{R} \otimes_{A} K$ [2, Proposition 7.1], $\& \approx \operatorname{Hom}_{A}(N, N)$ where $N$ is a finitely generated reflexive $A$-module [3, Proposition 4.2] and also $\mathfrak{R}_{\mathfrak{p}} \approx \operatorname{Hom}_{\boldsymbol{A}_{\mathfrak{p}}}\left(F_{\mathfrak{p}}, F_{\mathfrak{p}}\right)$ for each prime $p$.

Thus there exists, for each $\mathfrak{p}$, a reflexive ideal $I^{\mathfrak{p}}$ in $A_{\mathfrak{p}}$ such that $N_{\mathfrak{p}} \approx I^{\mathfrak{p}} \otimes_{A_{\mathfrak{p}}} F_{\mathfrak{p}}$. Therefore $N$ is in $M$ and $\mathfrak{R}$ is the image of $N$.

So the exactness of the sequence is proven.

We see, then, that the kernel in which we are interested, of the $\operatorname{map} \theta$ from $\mathfrak{B}(A)$ to $\Pi \mathfrak{B}\left(A_{\mathfrak{p}}\right)$ is the cokernel of the map from $\boldsymbol{W} / \mathbf{C}$ to $M / P$. We will look now at some applications of this characterization of the kernel.

We will show first of all that the kernel of $\theta$ is a torsion group, and prove from this that $\mathfrak{B}(A)$ is a torsion group. This is in fact a special case of a known theorem, by A. Grothendieck [6, §2] which states that the Brauer group of any commutative ring is torsion. Grothendieck's proof is very sophisticated, using the methods of étale cohomology. The proof offered here is elementary, and has some interesting aspects, and is therefore included in spite of its lack of generality.

THEOREM 2. The kernel of $\theta$ is a torsion group.

Proof. We must show that if $\mathbb{R}$ is in the kernel of $\theta$, then $\mathscr{Q} \otimes \mathbb{R}$ $\otimes \cdots \otimes \mathfrak{Q}(r$ times $)$ is the endomorphism ring of a projective $A$ module for some $r$.

We have seen that if $\mathfrak{R}_{\mathfrak{p}}$ is trivial in $\mathfrak{B}\left(A_{\mathfrak{p}}\right)$ for each $\mathfrak{p}$, then

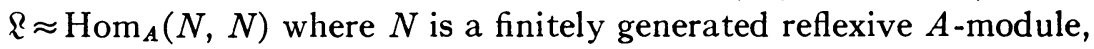


and the localization $N_{\mathfrak{p}}$ is isomorphic to $I^{\mathfrak{b}} \otimes_{A_{\mathfrak{p}}} F_{\mathfrak{p}}$ where $I^{\mathfrak{b}}$ is a reflexive ideal in $A_{\mathfrak{p}}$, and $F_{\mathfrak{p}}$ is a finitely generated free $A_{\mathfrak{p}}$-module. Let $r$ be the rank of $F_{\mathfrak{p}}$ over $A_{\mathfrak{p}}$. The rank is independent of the prime $\mathfrak{p}$, for in each case $r=\operatorname{dim}_{K}\left(F_{\mathfrak{p}} \otimes_{A_{\mathfrak{p}}} K\right)$. Then for each $\mathfrak{p}, N_{\mathfrak{p}}$ is isomorphic to the direct sum of $I^{\mathfrak{p}}, r$ times. Thus the $r$ th exterior power of $N_{\mathfrak{p}}$, $\Lambda_{A_{\mathfrak{p}}}^{\tau}\left(N_{\mathfrak{p}}\right)$, is isomorphic to the direct sum

$$
\bigoplus_{q_{1}+\cdots+q_{r}=r ; 0 \leq q_{i} \leq r}\left(\Lambda^{q_{1}(I \mathfrak{p})} \underset{A_{\mathfrak{p}}}{\otimes} \Lambda^{q_{2}}\left(I^{\mathfrak{p}}\right) \underset{A_{\mathfrak{p}}}{\otimes} \cdots \underset{A_{\mathfrak{p}}}{\otimes} \Lambda^{q_{r}\left(I^{\mathfrak{p}}\right)}\right)
$$

$[4$, p. 74$]$, and the double dual, $\Lambda^{r}\left(N_{\mathfrak{p}}\right)^{* *}$ is isomorphic to the direct sum

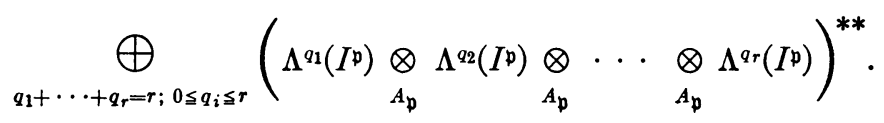

Now if $q_{i}>1, \Lambda^{q_{i}}\left(I^{\mathfrak{p}}\right)$ is a torsion module, for $\Lambda_{A_{\mathfrak{p}}}^{q_{i}}\left(I^{\mathfrak{p}}\right) \otimes_{A_{\mathfrak{p}}} K$ $\approx \Lambda_{K}^{q_{i}}\left(I^{\mathfrak{p}} \otimes_{A_{\mathfrak{p}}} K\right)$ (since exterior powers commute with localization). But $I^{\mathfrak{p}} \otimes_{A_{\mathfrak{p}}} K \approx K$, so $\Lambda_{K}^{q_{i}}\left(I^{\mathfrak{p}} \otimes_{A_{\mathfrak{p}}} K\right)$ is 0 for $q_{i}>1$. Thus $\Lambda_{K}^{q_{\mathfrak{i}}}\left(I^{\mathfrak{p}}\right)$ $\otimes \cdots \otimes \Lambda^{q_{r}}\left(I^{\mathfrak{p}}\right)$ is a torsion module for every term of the direct sum which contains an index $q_{i}>1$. Hence, for each $\mathfrak{p}, \Lambda^{r}\left(N_{\mathfrak{p}}\right)^{* *}$ is simply $\left(I^{\mathfrak{p}} \otimes I^{\mathfrak{p}} \otimes \cdots \otimes I^{\mathfrak{p}}\right)^{* *}$ ( $r$ times). We recall that this is the product $\left(I^{\mathfrak{p}}\right)^{r}$ in the group $W\left(A_{\mathfrak{p}}\right)$.

Now consider the $r$ th exterior power of $N, \Lambda_{A}^{r}(N)$. This is a module of rank 1, since $\Lambda_{A}^{r}(N) \otimes_{A} K \approx \Lambda_{A}^{\tau}(N) \otimes_{A} A_{\mathfrak{p}} \otimes_{A_{\mathfrak{p}}} K \approx \Lambda_{K}^{r}\left(N_{\mathfrak{p}} \otimes_{A_{\mathfrak{p}}} K\right)$ $\approx \Lambda_{K}^{r}\left(I^{\mathfrak{p}} \otimes_{A_{\mathfrak{p}}} F_{\mathfrak{p}} \otimes_{A_{\mathfrak{p}}} K\right) \approx \Lambda_{K}^{r}\left(F_{\mathfrak{p}} \otimes_{A_{\mathfrak{p}}} K\right) \approx K$ since $F_{\mathfrak{p}} \otimes_{A_{\mathfrak{p}}} K$ is a vector space over $K$ of dimension $r$ for each $\mathfrak{p}$. Thus $\Lambda_{A}^{r}(N)^{* *}$ is a reflexive $A$-module of rank 1 , and is therefore isomorphic to a reflexive ideal $J$ in $W(A)$. Now $\left(J^{-1} \otimes_{A} N^{r}\right)^{* *}=J^{-1} \circ N^{r}$ (considered as a product in $\boldsymbol{M}(A))$ is a projective $A$-module, since if we localize at any prime ideal $\mathfrak{p}$ of $A$ we have $\left(J^{-1} \circ N^{r}\right)_{\mathfrak{p}} \approx J_{\mathfrak{p}}^{-1} \circ N_{\mathfrak{p}}^{r}$ (in $\boldsymbol{M}\left(A_{\mathfrak{p}}\right)$ ) $\approx J_{\mathfrak{p}}^{-1} \circ\left(I^{\mathfrak{p}}\right)^{r} \circ F_{\mathfrak{p}}^{r} \approx J_{\mathfrak{p}}^{-1} \circ J_{\mathfrak{p}} \circ F_{\mathfrak{p}}^{r}$ which is a free $A_{\mathfrak{p}}$-module. Thus $N^{r}=J \circ P$ where $P$ is a finitely generated projective $A$-module. Now we see that $\Omega^{r}=\left(\operatorname{Hom}_{A}(N, N)\right)^{r} \approx \operatorname{Hom}_{A}\left(N^{r}, N^{r}\right) \approx \operatorname{Hom}_{A}(P, P)$, so that $\mathfrak{R}$ is a torsion element of the Brauer group $\mathfrak{B}(A)$.

Thus the kernel of $\theta$ has been shown to be a torsion group.

We will use this fact to demonstrate that for $A$ an integrally closed noetherian domain, the Brauer group $\mathfrak{B}(A)$ is itself a torsion group. We will show that for any element $\mathfrak{R}$ of the Brauer group there is an $n$ such that $\mathbb{R}^{n}$ is in the kernel of $\theta$. (It might be of interest here to note that the truth of this latter statement does not depend on any conditions on the commutative ring $A$.) 
First we will show that a local isomorphism of elements of the Brauer group can be extended to a neighborhood.

LeмmA. Let $\mathfrak{R}_{1}$ and $\mathfrak{R}_{2}$ be A-algebras, $\mathfrak{R}_{1}$ finitely presented as an $A$ module, and suppose that for some prime ideal $\mathfrak{p}$ of $A$ there is an $A_{p-}$ algebra homomorphism $h: \mathfrak{R}_{1_{p}} \rightarrow \mathfrak{R}_{2 \mathrm{p}}$. Then there is a neighborhood $D(f)$ of $p$ to which $h$ can be extended. If $h$ is an isomorphism then there is a neighborhood on which the extended map is an isomorphism.

Proof. Since $\Omega_{1}$ is finitely presented there is a map $\bar{h}$ of $A$-modules $\mathfrak{l}_{1}$ and $\mathfrak{R}_{2}$ which when localized at $\mathfrak{p}$ gives the $A_{\mathfrak{p}}$-module map $h$. We want to show that there is a neighborhood $D(f)$ of $\mathfrak{p}$ such that $\bar{h}_{f}: \Omega_{1 f} \rightarrow \Omega_{2 f}$ is a homomorphism of $A_{f}$-algebras. This fact is a consequence of the following easily verified proposition: if $M$ is a finitely generated $A$-module and $g: M \rightarrow N$ is a homomorphism of $A$-modules which becomes trivial when localized at a prime ideal $\mathfrak{p}$ of $A$, then there is a neighborhood $D(f)$ of $\mathfrak{p}$ such that $g$ becomes trivial when localized at $f$. We apply this proposition to the diagram of $A$-modules

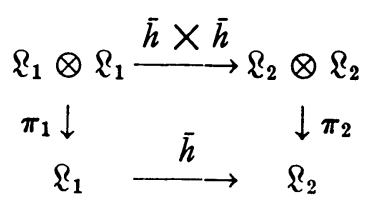

where $\pi_{1}$ and $\pi_{2}$ are the homomorphisms defining multiplication in $\mathfrak{R}_{1}$ and $\Omega_{2}$ respectively. By hypothesis this diagram commutes when localized at the prime ideal $\mathfrak{p}$. If we let the role of $g$ be played by $\pi_{2}(\bar{h} \times \bar{h})-\bar{h} \pi_{1}$, we see that the diagram must commute when localized at some $f$ not in p. For this $f$ the map $\bar{h}_{f}$ is an $A_{f}$-algebra homomorphism.

Now suppose that $h$ is an isomorphism. Consider the exact sequence

$$
0 \rightarrow Q \stackrel{i}{\rightarrow} \mathfrak{R}_{1 f} \stackrel{\bar{h}_{f}}{\rightarrow} \Omega_{2 f} \stackrel{j}{\rightarrow} R \rightarrow 0
$$

where $Q$ is the kernel and $R$ the cokernel of $\bar{h}_{f}$. Localizing at $\mathfrak{p}$, we have $i_{p}=j_{p}=0$. Then, invoking the same proposition as was used above, we see that there is a neighborhood of $p$ contained in $D(f)$ for which the localizations of $i$ and $j$ are trivial, and hence for which the localization of $\bar{h}_{f}$ is an isomorphism.

THEOREM 3. If $A$ is an integrally closed noetherian domain, $\mathfrak{B}(A)$ is a torsion group. 
Proof. Let $\ell$ be a central separable algebra over $A$, and $\mathfrak{p}$ a prime ideal in $A$. Then [2, Corollary 6.4] $\mathfrak{R}_{\mathfrak{p}}$ is split by a Galois extension $S$ of $A_{\mathfrak{p}}$; i.e., $\mathfrak{R}_{\mathfrak{p}} \otimes_{A_{\mathfrak{p}}} S \approx \operatorname{Hom}_{\mathcal{S}}(M, M)$ where $M$ is a finitely generated projective faithful $S$-module.

Since $S$ is a semilocal ring, there is an exact sequence $0 \rightarrow H^{2}(G, U(S))$ $\rightarrow \mathfrak{B}\left(A_{\mathfrak{p}}\right) \rightarrow \mathfrak{B}(S)$ where $G$ is the Galois group of $S$ over $A_{\mathfrak{p}}$ and $U(S)$ is the group of units of $S[2, \mathrm{p} .386]$. Now since the image of the class of $\mathfrak{R}_{\mathfrak{p}}$ in $\mathfrak{B}(S)$ is trivial, the class of $\mathfrak{R}_{\mathfrak{p}}$ comes from an element in $H^{2}(G, U(S))$, a group all of whose elements have finite order. Therefore the class of $\mathfrak{R}_{\mathfrak{p}}$ has finite order in $\mathfrak{B}\left(A_{\mathfrak{p}}\right)$; i.e., there is an integer $n$ and a free module $F$ such that $\left(\Omega_{\mathfrak{p}}\right)_{\mathfrak{p}} \approx \operatorname{Hom}_{A_{\mathfrak{p}}}\left(F_{\mathfrak{p}}, F_{\mathfrak{p}}\right)$. Then from the Lemma above, we see that there is a neighborhood $D\left(f_{\mathfrak{p}}\right)$ of $\mathfrak{p}$ such that $\left(\Omega_{f_{p}}\right)_{\mathfrak{p}} \approx \operatorname{Hom}_{A_{f \mathfrak{p}}}\left(F_{f_{\mathfrak{p}}}, F_{f_{\mathfrak{p}}}\right)$. Since Spec $A$ is covered by a finite number of neighborhoods $D\left(f_{\mathfrak{p}}\right)$, we can choose such a finite number and let $n$ be the largest of the $n_{\mathfrak{p}}$ 's. Then $\left(\mathfrak{Q}^{n}\right)_{\mathfrak{p}}$ is trivial in $\mathfrak{B}\left(A_{\mathfrak{p}}\right)$ for for every prime ideal $\mathfrak{p}$ of $A$. Thus $\mathfrak{R}^{n}$ belongs to the kernel of $\theta$ and therefore $\mathbb{R}$ has finite order.

Another application of our characterization of the kernel of $\theta$ is the fact that it is isomorphic to a subgroup of the first cohomology group of the sheaf of Cartier divisors. We will define $w$, the sheaf of divisors and $\mathfrak{e}$, the sheaf of Cartier divisors, whose global sections are respectively $\boldsymbol{W}$ and $\boldsymbol{C}$.

Let $D(f)$ be a basic open set of Spec $A$. We define $W(D(f))$ to be $W\left(A_{f}\right)$, the group of finitely generated reflexive $A_{f}$-submodules of $K$. Since for each $f$ in $A, A_{f}$ is an integrally closed noetherian domain, we may use the previously noted characterization of $w(D(f))$ as the free abelian group generated by the minimal prime ideals of $A_{f}$. If $D(f) \supset D(g)$ then $A_{f} \subset A_{0}$. In this case the minimal primes of $A_{o}$ are precisely the ideals $A_{\boldsymbol{g}} \cdot \mathfrak{p}$ where $\mathfrak{p}$ is a minimal prime in $A_{f}$ which does not contain $g$. Thus we can define the restriction map $\rho_{g}^{f}$ of $\Psi(D(f))$ onto $W(D(G))$ by sending a minimal prime $\mathfrak{p}$ of $A_{f}$ which does not contain $g$ onto $A_{\sigma} \cdot \mathfrak{p}$, and the others on to 0 . The map thus defined may also be expressed as $\rho_{o}^{f}\left(M^{f}\right)=M^{f} \otimes_{A_{f}} A_{o}$, the localization of $M^{f}$ at $g$, for every $M^{f}$ in $w(D(f))$.

It is readily verified that $w$ thus defined on a basis of Spec $A$ gives rise to a sheaf of groups, and that the global section $\Gamma(\operatorname{Spec} A, W)$ is $W$. We notice also that the restriction map is an epimorphism, so that the sheaf $W$ is flasque.

Now we can construct the subsheaf $\mathfrak{e}$ of $\mathfrak{W}$ by defining $\mathcal{C}(D(f))$ to be the group of finitely generated projective $A_{f}$-submodules of $K$. If $M^{f}$ is in $\mathcal{C}(D(f))$ and $D(f) \supset D(g)$ then $\rho_{\theta}^{f}\left(M^{f}\right)=M^{f} \otimes_{A_{f}} A_{0}$ is 
projective over $A_{g}$. Thus $\rho_{o}^{f}$ maps $\mathcal{C}(D(f))$ into $\mathfrak{e}(D(g))$. We need only verify that if $D(f)=\bigcup_{i \in I} D\left(f_{i}\right)$ and $M^{f_{i}} \in \mathcal{e}\left(D\left(f_{i}\right)\right)$ such that $M_{f_{i} f_{j}}^{f_{i}}$ $=M_{f_{i} f_{j}}^{f_{j}}$ for every $i$ and $j$ in $I$, then the reflexive module $M^{f} \in W(D(f))$ which has the property that $M_{f_{i}}^{f}=M^{f_{i}}$ for each $i$ in $I$ is projective. This follows from the fact that for every prime ideal $p$ in $D(f)$ (not necessarily minimal), $\mathfrak{p}$ is in some $D\left(f_{i}\right)$. Then $M_{\mathfrak{p}}^{\mathfrak{f}}=M_{\mathfrak{p}}^{f_{i}}$ which is a free module over $A_{\mathfrak{p}}$. Thus $M_{\mathfrak{p}}^{f}$ is free for every prime ideal $\mathfrak{p}$ in $A_{f}$ and $M^{f}$ is therefore projective. Thus $\mathcal{C}$ is a sheaf. It is clear that $\Gamma(\operatorname{Spec} A, \mathfrak{e})=\boldsymbol{C}$.

Let us look at the exact sequence of sheaves $0 \rightarrow \mathfrak{C} \rightarrow W \rightarrow W / \mathfrak{C} \rightarrow 0$ (where $w / \mathcal{C}$ is of course the quotient sheaf) giving rise to the exact sequence

$$
\begin{aligned}
& 0 \rightarrow \Gamma(\operatorname{Spec} A, \mathfrak{e}) \rightarrow \Gamma(\operatorname{Spec} A, w) \\
& \rightarrow \Gamma(\operatorname{Spec} A, W / \mathcal{C}) \rightarrow H^{1}(\operatorname{Spec} A, \mathfrak{e}) \rightarrow 0
\end{aligned}
$$

(since $W$ is flasque) which is the same as

$$
0 \rightarrow C \rightarrow W \rightarrow \Gamma(\operatorname{Spec} A, \mathfrak{W} / \mathcal{C}) \rightarrow H^{1}(\operatorname{Spec} A, \mathfrak{e}) \rightarrow 0 .
$$

We will show that there is an injection $\alpha$ of $M / P$ into $\Gamma(\operatorname{Spec} A, W / \mathfrak{C})$. To begin with, we define a map $\bar{\alpha}$ from $\boldsymbol{M}$ to $\Gamma(\operatorname{Spec} A, W / \mathcal{C}$ ) as follows: if $M$ is in $\boldsymbol{M}$, then for each prime ideal $\mathfrak{p}$ of $A, M_{\mathfrak{p}} \approx I^{\mathfrak{p}} \otimes_{A_{\mathfrak{p}}} F_{\mathfrak{p}}$ where $I^{\mathfrak{p}}$ is a reflexive ideal in $A_{\mathfrak{p}}$ and $F_{\mathfrak{p}}$ is a free finitely generated $A_{\mathfrak{p}}$-module. Let $\left[I^{\mathfrak{p}}\right]$ be the class of $I^{\mathfrak{p}}$ in $\mathfrak{W}_{\mathfrak{p}} / \mathfrak{C}_{\mathfrak{p}}$. Then let $\bar{\alpha}(M)$ be $\left\{\left[I^{\mathfrak{p}}\right]\right\}_{\mathfrak{p} \in \operatorname{Spec} A}$. This map is well defined, for suppose $M_{\mathfrak{p}} \approx I^{\mathfrak{p}} \otimes_{A_{\mathfrak{p}}} F_{\mathfrak{p}} \approx J^{\mathfrak{p}} \otimes_{A_{\mathfrak{p}}} G_{\mathfrak{p}}$ where $I^{\mathfrak{p}}$ and $J^{\mathfrak{p}}$ are reflexive, $F_{\mathfrak{p}}$ and $G_{\mathfrak{p}}$ free. Let $\left(I^{\mathfrak{p}}\right)^{*}$ be the inverse of $I^{\mathfrak{p}}$ in the group of finitely generated reflexive $A_{\mathfrak{p}}$-submodules of $K$, and let $\left(J^{\mathfrak{p}}\right)^{*}$ be the inverse of $J^{\mathfrak{p}}$. Then under the multiplication in this group $M_{\mathfrak{p}} \approx I^{\mathfrak{p}} \circ F_{\mathfrak{p}} \approx J^{\mathfrak{p}} \circ G_{\mathfrak{p}}$, so $\left(I^{\mathfrak{p}}\right)^{*} \circ I^{\mathfrak{p}} \circ F_{\mathfrak{p}} \approx\left(I^{\mathfrak{p}}\right)^{*} \circ J^{\mathfrak{p}} \circ G_{\mathfrak{p}}$. Thus $\left(I^{\mathfrak{p}}\right)^{*} \circ J^{\mathfrak{p}}$ is isomorphic to a direct summand of a free module and is therefore projective, hence, in this case, free. Thus $\left(I^{\mathfrak{p}}\right)^{*} \circ J^{\mathfrak{p}}$, being a free ideal, can only be $A_{\mathfrak{p}}$, and $J^{\mathfrak{p}}$ is isomorphic to $I^{\mathfrak{p}}$.

Now we need to verify that $\left\{\left[I^{\mathfrak{p}}\right]\right\}_{\mathfrak{p} \in \operatorname{Spec} A}$ is indeed an element of $\Gamma(\operatorname{Spec} A, W / \mathcal{C})$. We must show that for every prime ideal $\mathfrak{p}$ of $A$, there is a neighborhood $D(f)$ of $\mathfrak{p}$ and an element $\left[I^{f}\right]$ of $\mathscr{W}(D(f)) / \mathfrak{C}(D(f))$ such that the localization $I_{q}^{f} \approx I^{q}$ for each prime $\mathfrak{q}$ in $D(f)$. For fixed prime $\mathfrak{p}, I^{\mathfrak{p}}$ is the localization at $\mathfrak{p}$ of some reflexive ideal $\bar{I}$ of $A$, so $M_{\mathfrak{p}} \approx \bar{I}_{\mathfrak{p}} \otimes_{A_{\mathfrak{p}}} F_{\mathfrak{p}}$. Let $\bar{I}^{*}$ be the inverse of $\bar{I}$ in $W$. Then $\left(\bar{I}^{*} \circ M\right)_{\mathfrak{p}} \approx F_{\mathfrak{p}}$, a free $A_{\mathfrak{p}}$-module. Hence there is a neighborhood $D(f)$ of $\mathfrak{p}$ such that $\left(\bar{I}^{*} \circ M\right)_{f} \approx F_{f}$, or $M_{f} \approx I_{f} \otimes_{A_{f}} F_{f}$. Letting $I^{f}=\bar{I}_{f}$ we see that the required condition is fulfilled. 
Since $\left(M \otimes_{A} N\right)_{\mathfrak{p}}^{* *}=M_{\mathfrak{p}} \otimes_{A} N_{\mathfrak{p}}$ for all $M$ and $N$ in $M$ and $\mathfrak{p}$ in Spec $A$, we see that $\bar{\alpha}$ is a homomorphism of groups.

TheOREM 4. The map $\bar{\alpha}$ defines a monomorphism $\alpha: M / P$ $\rightarrow \Gamma(\operatorname{Spec} A, w / \mathfrak{C})$ so that we have a commutative diagram

$$
\begin{aligned}
& 0 \rightarrow W / C \rightarrow \Gamma(\operatorname{Spec} A, \mathfrak{W} / \mathcal{C}) \rightarrow H^{1}(\operatorname{Spec} A, \mathfrak{e}) \rightarrow 0 \\
& \begin{array}{c}
\| \\
0 \rightarrow W / C \longrightarrow
\end{array} \stackrel{M / P \rightarrow \mathfrak{B}(A) \stackrel{\theta}{\rightarrow} \Pi \mathfrak{B}\left(A_{\mathfrak{p}}\right)}{\longrightarrow} \\
& \uparrow \\
& 0
\end{aligned}
$$

and an induced monomorphism from the kernel of $\theta$ to $H^{1}(\operatorname{Spec} A, \mathfrak{e})$.

Proof. It is easy to see that $P$ is in the kernel of $\bar{\alpha}$. For $I^{\mathfrak{p}}$ is a free $A_{\mathfrak{p}}$-module for each $\mathfrak{p}$ if and only if $M_{\mathfrak{p}}$ is a free $A_{\mathfrak{p}}$-module for each $\mathfrak{p}$; i.e., if and only if $M$ is a projective $A$-module. Thus $\bar{\alpha}$ induces a monomorphism $\alpha: M / P \rightarrow \Gamma(\operatorname{Spec} A, \mathfrak{W} / \mathcal{C})$. It is clear from the definition of $\bar{\alpha}$ that the diagram commutes. Thus there is a homomorphism defined from the image of $\boldsymbol{M} / \boldsymbol{P}$ in $\mathfrak{B}(A)$ into $H^{1}(\operatorname{Spec} A, \mathfrak{C})$. A small amount of diagram chasing verifies that this homomorphism is an injection.

Thus we see that the kernel of $\theta$ is isomorphic to a subgroup of $H^{1}$ (Spec $\left.A, \mathfrak{e}\right)$, and, in fact, in view of the earlier result, to a subgroup of the torsion subgroup of $H^{1}(\operatorname{Spec} A, \mathfrak{C})$.

Some particular applications of this result are readily arrived at in the case where $H^{1}(\operatorname{Spec} A, \mathcal{e})$ is trivial.

Corollary 4.1. If $A$ is a semilocal domain, then $\theta$ is a monomorphism.

Proof. Since $A$ is a semilocal ring, every projective fractionary ideal is principal $[2$, p. 377 , Remark]. Thus if $D(f) \supset D(g)$ the restriction map $\rho_{g}^{f}: \mathfrak{C}(D(f)) \rightarrow \mathfrak{C}(D(g))$ is an epimorphism, so that $\mathfrak{e}$ is flasque. Thus $H^{1}(\operatorname{Spec} A, \mathfrak{e})$ is trivial, and so is the kernel of $\theta$.

Corollary 4.2. If $A$ is a local unique factorization domain, then $\theta$ is a monomorphism.

Proof. Since $A$ is a local unique factorization domain, every reflexive fractionary ideal is projective. Hence $C=W, \mathfrak{C}=W$ and $H^{1}(\operatorname{Spec} A, \mathrm{e})$ is therefore trivial.

Corollary 4.3. Let $A$ have the property that $A_{\mathfrak{p}}$ is a unique factoriza- 
tion domain for all but a finite number of prime ideals $\mathfrak{p}$. Then $\theta$ is a monomorphism.

Proof. Let $\mathfrak{p}_{1}, \cdots, \mathfrak{p}_{n}$ be all the prime ideals for which $A_{\mathfrak{p}}$ is not a unique factorization domain. Let $S=A-\bigcup_{i=1}^{n} \mathfrak{p}_{i}$. Then the ring $A_{S}$ is a semilocal domain, with $n$ maximal ideals. Suppose $\mathscr{R}$ is in the kernel of $\theta$. Then we know that $\& \approx \operatorname{Hom}_{A}(M, M)$ where $M$ is in $\boldsymbol{M}$. From Corollary 4.1 we see that this implies that $M_{S} \approx I_{S} \otimes_{A_{S}} F_{S}$, where $F_{S}$ is a free finitely generated $A_{S}$-module (since from $[2$, p. 377 , Remark] we know that every finitely generated projective $A_{S^{-}}$ module is free) and $I_{S}$ is a reflexive ideal in $A_{S}$, which may be taken to be the localization at $S$ of a reflexive ideal $I$ in $A$. Now if $I^{*}$ is the inverse of $I$ in $W$ we would like to show that $I^{*} \circ M$ is a projective $A$-module. If we localize at any prime $\mathfrak{p}$ of $A$ which is not one of the $\mathfrak{p}_{i}, i=1, \cdots, n$, then $\left(I^{*} \circ M\right)_{\mathfrak{p}}$ is free since $A_{\mathfrak{p}}$ is a unique factorization domain. On the other hand, for each of the $\mathfrak{p}_{i}, i=1, \cdots, n$, $\left(I^{*} \circ M\right)_{\mathfrak{p}_{i}} \approx F_{\mathfrak{p}_{i}}$ and is therefore free. Thus $I^{*} \circ M$ is projective, and $\mathfrak{Z}$ is seen to be trivial.

CoROllaRY 4.4. If $A$ is an integrally closed affine geometric ring of Krull dimension 2, then the kernel of $\theta$ is trivial.

Proof. In this case, $A$ satisfies the hypothesis of Corollary 4.3.

\section{BIBLIOGRAPHY}

1. B. Auslander, The Brauer group of a ringed space, J. Algebra 4 (1966), 220-273. MR 33 \#7362.

2. M. Auslander and O. Goldman, The Brauer group of a commutative ring, Trans. Amer. Math. Soc. 97 (1960), 367-409. MR 22 \#12130.

3. — Maximal orders, Trans. Amer. Math. Soc. 97 (1960), 1-24. MR 22 \#8034.

4. N. Bourbaki, Algèbre, Chapitre 3, Actualités Sci. Indust., no. 1044, Hermann, Paris, 1948. MR 10, 231.

5. — Algèbre commutative, Chapitre 7, Hermann, Paris, 1965.

6. A. Grothendieck, Groupes de Brauer, Séminaire Bourbaki 1964/65, Exposé 290, Benjamin, New York, 1966. MR 33 \#54201.

Wellesley College, Wellesley, Massachusetts 02181 